\title{
Case Report: Treatment of Widespread Nodular Post kala-Azar Dermal Leishmaniasis with Extended-Dose Liposomal Amphotericin B in Bangladesh: A Series of Four Cases
}

\author{
Ariful Basher, ${ }^{1 \star}$ Shomik Maruf, ${ }^{2}$ Proggananda Nath, ${ }^{1}$ Md Golam Hasnain, ${ }^{3}$ Muhammod Abdul Mukit, ${ }^{1}$ Azim Anuwarul, ${ }^{1}$ \\ Fatima Aktar, ${ }^{2}$ Rupen Nath, ${ }^{2}$ AFM Akhtar Hossain, ${ }^{4}$ Abul Hasnat Milton, ${ }^{3}$ Dinesh Mondal, ${ }^{2}$ \\ Abul Khair Mohammad Shamsuzzaman, ${ }^{5}$ Ridwanur Rahman, ${ }^{6}$ and M. Abul Faiz ${ }^{7}$ \\ ${ }^{1}$ Infectious and Tropical Medicine Department, Mymensingh Medical College and Hospital (MMCH), Mymensingh, Bangladesh; ${ }^{2}$ Nutrition and \\ Clinical Services Division (NCSD), International Centre for Diarrhoeal Disease Research, Bangladesh (icddr,b), Dhaka, Bangladesh; ${ }^{3}$ Centre for \\ Clinical Epidemiology and Biostatistics (CCEB), School of Medicine and Public Health, Faculty of Health and Medicine, The University of Newcastle \\ $(U o N)$, New South Wales (NSW), Australia; ${ }^{4}$ National Kala-azar Elimination Program (NKEP), Directorate General Health Services (DGHS); \\ Ministry of Health and Family Welfare (MoHFW), Government of Bangladesh (GoB), Dhaka, Bangladesh; ${ }^{5}$ Disease Control Unit, Directorate \\ General Health Services (DGHS), Ministry of Health and Family Welfare (MoHFW), Government of Bangladesh (GoB), Dhaka, Bangladesh; \\ ${ }^{6}$ Department of Medicine, Shaheed Suhrawardi Medical College and Hospital (SSMCH), Dhaka, Bangladesh; ${ }^{7}$ Dev Care Foundation, \\ Dhaka, Bangladesh
}

\begin{abstract}
Post kala-azar dermal leishmaniasis (PKDL) is a skin manifestation which usually appears after visceral leishmaniasis. It is now proved that PKDL patients serve as a reservoir for anthropometric leishmanial transmission. Hence, to achieve the kala-azar elimination target set by the World Health Organization in the Indian Subcontinent, PKDL cases should be given priority. The goal of treatment for PKDL should be early reepithelizlization and rapid cure, but unfortunately this has been difficult to achieve, especially for patients with severe lesions. Therefore, we describe here four cases of PKDL who had widespread nodular and macular lesions and were treated with two cycles of LAmB doses with $20 \mathrm{mg} / \mathrm{kg}$ body weight divided into four equal doses (each dose contains $5 \mathrm{mg} / \mathrm{kg}$ ) administered every alternate day. This treatment schedule achieved $100 \%$ treatment success with the minimal safety concern.
\end{abstract}

\section{BACKGROUND}

Post Kala-azar Dermal Leishmaniasis (PKDL) is a sequela of visceral leishmaniasis (VL) which generally appears after the patients have been cured of VL. However, there are reports of patients suffering from PKDL without any prior history of VL. ${ }^{1}$ PKDL appears as a macular, papular, or nodular rash or a combination of these, generally starting from the face but may start from any other part of the body. It involves the arm, trunk, abdomen, back, gluteal region, thigh, and leg. However, it can also affect the conjunctival, nasal, oral and genital mucosa, hands, and feet. ${ }^{2,3}$ Other than the skin lesions, the patients of PKDL usually do not have other problems and as a result are not interested in taking treatment. This poses a problem for the rest of the community as untreated cases are now proven to be reservoirs for harboring the parasite, thereby, propagating the infection if there is the absence of early case detection and management. ${ }^{3,4}$

The type of drug treatment of PKDL is now considered to be the most important issue regarding case management. Previously, sodium stibogluconate (SSG) was the only treatment option available but it is now being phased out because of its toxicity and poor compliance. ${ }^{5}$ Currently, miltefosine (MF), amphotericin B deoxycholate, and liposomal amphotericin B (LAmB) are recommended by the World Health Organization as treatment options for PKDL. ${ }^{2}$ A national program of Bangladesh recommends MF for 12 weeks as first-line option and LAmB $(20 \mathrm{mg} / \mathrm{kg}$ body-weight into four equally divided doses on every alternate day) as an alternative treatment option for PKDL. ${ }^{6}$ However, in Bangladesh, a short course treatment with two doses of LAmB (5 mg/kg per dose) per week for 3 weeks proved to be successful to some extent and

*Address correspondence to Ariful Basher, Infection and Tropical Medicine, Mymensingh Medical College Hospital (MMCH), Mymensingh, Bangladesh. E-mail: ariful.dr@gmail.com follow-up after 12 weeks of treatment shows $34 \%$ complete reepithelization. ${ }^{2}$ Although the efficacy of MF is $93 \%$, it seemed to be ineffective (decreased efficacy and development of drug resistance) in many cases. ${ }^{7,8}$ By contrast, low dose LAmB has $90 \%$ efficacy with initial response, ${ }^{2}$ highlighting the fact that larger doses could be used to prompt early reepithelization and rapid cure, especially for patients with severe lesions.

Here, we describe four cases of PKDL who had widespread nodular and macular lesions and were treated with two cycles of LAmB doses with $20 \mathrm{mg} / \mathrm{kg}$ body weight divided into four equal doses (each dose contains $5 \mathrm{mg} / \mathrm{kg}$ ) administered every alternate day.

\section{CASE PRESENTATION}

Case 1. A 60-year-old male from Kapasia subdistrict under Gazipur district was admitted in the Surja Kanta Kala-azar Research Center (SKKRC) with complaints of the progressive nonitching nodular type of skin rash for the last 5 years. He had a previous history of VL 30 years ago and was treated with SSG. He stated that initially, he had a nonitching macular shaped rash for 23 years on his chest and back. Despite the presence of the rash, he did not seek treatment as it was not causing any physical problems. Over the last 5 years, he started to develop a nodular type of rash with his chin being the first site affected. He went to a local private doctor who prescribed topical and oral medications. However, the medication did not provide relief and his condition worsened continually. Last year, the disease process became aggressive and the rash spread quickly to both of his arms, feet, genitalia, and nose. He went to a nearby subdistrict hospital and they referred him to the SKKRC as a suspected case of PKDL for further evaluation and confirmation. On examination, hospital physicians found a macular rash on his chest and back along with nodular lesions on his arm, hand, feet, genitalia and nose. Hospital physicians also found nasal bone erosion and ulceration in the scrotum. General and systemic 
examinations revealed no other abnormality, particularly no hepatosplenomegaly. The skin sensation was intact, including on sites of the rash and nodular lesions. After a physical examination, the hospital laboratory technician performed rK39 strip test which was positive. Finally, to confirm the diagnosis of PKDL, microscopic examination of slit skin smear and PCR (polymerase chain reaction) were performed and both were found to be positive (Figures 1 and 2). A 3-mm skin punch biopsy material was used for PCR using Taqman primer and probe: 5'-GCGACGTCCGTGGAAAGAA-3', 5' -GGCGGGTACACATTAGCAGAA-3', and reporter (fluorescein amidite): 5'-CAACGCGTATTCCC-3' (Applied Biosystems Inc., Foster City, CA). ${ }^{1,9}$ The LAmB treatment was initiated according to national guidelines, at a dose of $20 \mathrm{mg} / \mathrm{kg}$ into four divided doses every alternate day. Before starting treatment, his haematological, electrolytes, renal, and hepatic markers were assessed and all were found to be within normal limits (Table 1). After completion of the first cycle, the patient displayed noticeable improvement but not complete recovery. Physicians prescribed another cycle, which was given 28 days after the completion of the first cycle as the bioavailability of LAmB sustains for 28 days and its half-life is 7 days. ${ }^{12}$ The treatment schedule and dose were same as the first cycle. Oncompletion of the second cycle his haematological, electrolytes, renal, and hepatic markers were assessed again and all were found to be within normal or near normal limits (Table 1). A follow-up was done 28 days after completion of the second cycle and a progressive pattern of the resolution was noted. A slit skin smear microscopic examination and PCR were also conducted; both tests were negative (Figure 1). Before treatment and after treatment pictures are shown in Figure 1. During treatment, he only experienced fever with shivering. No other adverse event was noted.

Case 2. A 40-year-old male from Trishal subdistrict under Mymensingh district, the most endemic district for $V L$ in Bangladesh, had a previous history of VL 7 years ago and was treated with MF. He complained of having a macular type of lesion appear on his face and upper arms about 5 years ago. The disease progressed to nodular lesions on his chin, cheek, lip, tongue, hand, feet, and buttock over the last 2 years. Initially, he had ignored the macular lesions and did not seek treatment. However, at the appearance of the nodular lesions, he sought treatment from a traditional healer but his condition did not improve. One of his neighbors, who also suffered from PKDL, advised him to go to the SKKRC for disease confirmation and treatment. At SKKRC, physical examination

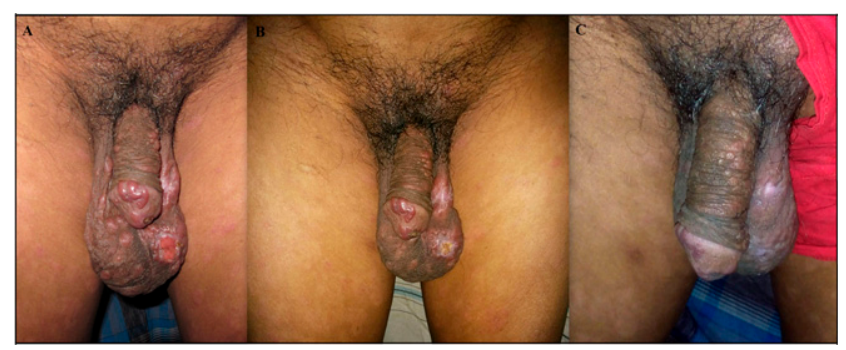

Figure 1. (A) Before treatment condition: nodular and ulcerative lesion; (B) Condition after first cycle: decreasing size of nodules and healing of ulcer; (C) Condition after complete treatment: disappearance of nodules and scars, only some scars are present. This figure appears in color at www.ajtmh.org.

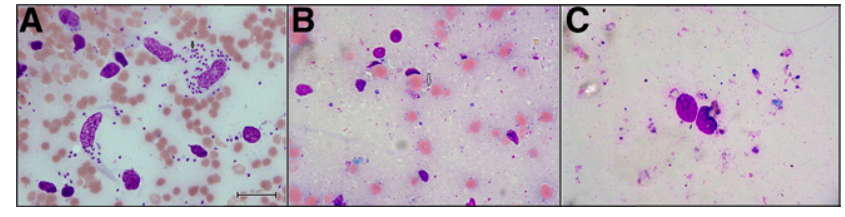

FIGURE 2. (A) Before treatment slit skin smear microscopy: presence of numerous amastigotes; (B) Slit skin smear microscopy after first cycle: presence of few amastigotes; (C) Slit skin smear microscopy after complete treatment: no amastigote. This figure appears in color at www.ajtmh.org.

results confirmed skin lesions mentioned on the previously mentioned affected sites. He did not have hepatosplenomegaly, and the lesions were nonitching with intact sensitivity. A positive rK39 strip test and microscopic examination of slit skin smear confirmed the diagnosis of PKDL. He was also treated with LAmB following the same protocol as Case 1. Before starting treatment, his haematological, electrolytes, renal, and hepatic markers were assessed and all were found to be within normal or near normal limits (Table 1). Follow-up slit skin smear examination was also conducted as Case 1 and was found to be negative. No treatment-related adverse event was documented during his treatment period.

Case 3. A 34-year-old male from Madargonj subdistrict of Jamalpur district, who had no previous history of VL despite having positive family history of VL, complained about a 5-year history of progressive nodular lesions. At first, nodules appeared on his chin and nose. He did not seek treatment initially but after 3 years when the lesions started to spread to other parts of the body, he contacted the village doctor. His condition did not improve and thus, he sought treatment from the subdistrict level hospital doctor who advised him to go to the SKKRC. At SKKRC, physical examination revealed nodular lesions on his chin, nose, arm, hand, buttock and leg but no other abnormality, particularly no organomegaly. Lesions were nonitching with intact skin sensitivity. Positive rK39 strip test, PCR, and slit skin smear examination established the presence of Leishmania parasite which ultimately diagnosed him as a case of PKDL. The treatment was initiated with LAmB at $20 \mathrm{mg} / \mathrm{kg}$ into four divided doses. After completion of the first cycle, he was advised to return after 28 days, where to follow-up revealed an improved condition but incomplete resolution of nodular lesions and persistent positive slit skin smear. Physicians decided to go for another cycle of LAmB treatment as Case 1 and Case 2 after taking into consideration their successful management. After completion of the second cycle, follow-up was conducted after 1 month where the progressive pattern of nodular lesion disappearance was observed with negative slit skin examination and PCR results. Three-point haematological, electrolytes, renal, and hepatic functions were assessed which were within normal or near normal limit (Table 1). He did not experience any adverse event during treatment.

Case 4. A 44-year-old male from Narsingdi sadar of Narsingdi district, which is now considered a nonendemic zone, complained of having a macular type rash for 15 years. He did not have any previous history of VL or positive family history. The macular rash first appeared on his chin, after which it spread to his back and upper arm. He did not seek treatment for it until two years ago when he noticed nodular lesions on his nose, face, back, and hand. He first consulted a 


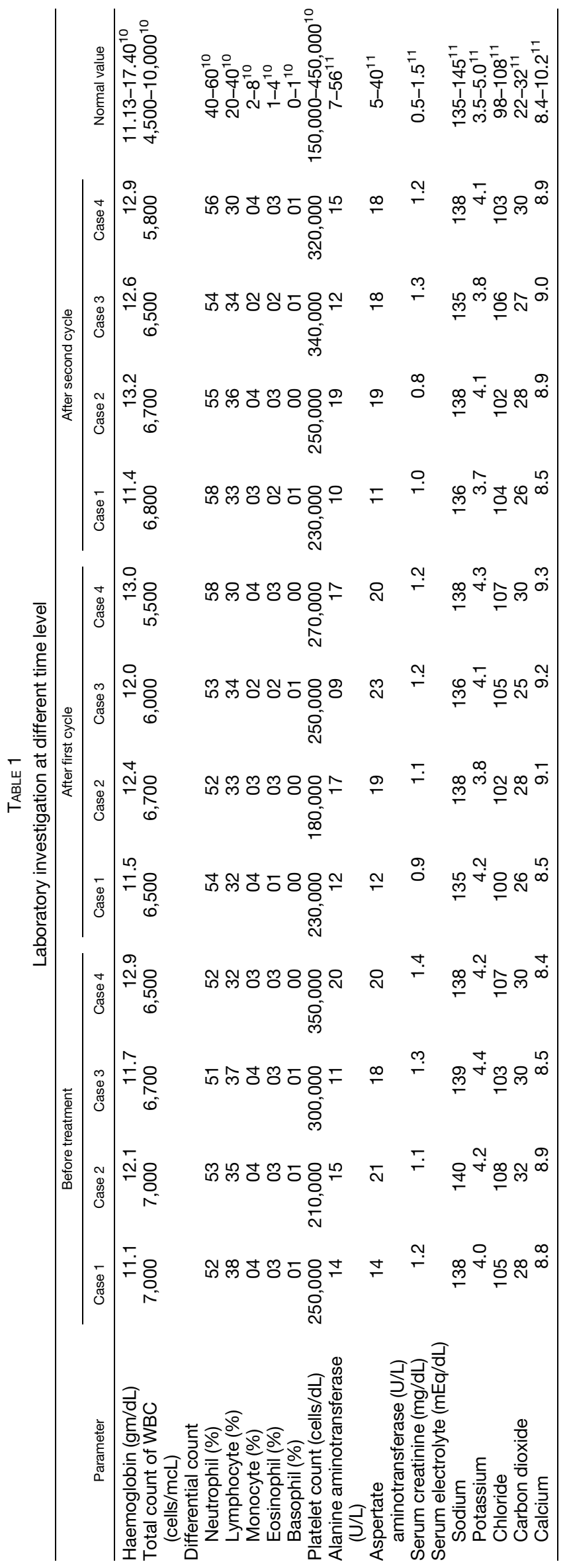


local medical graduate who referred him to the district hospital. A district hospital physician advised him to go to the SKKRC. Physical examination findings at SKKRC were consistent with PKDL presentation: rash on nose, face, back, and hand; absence of itching and intact sensitivity of lesions; and no hepatosplenomegaly. rK-39 strip test and slit skin smear microscopic examination revealed the parasitological confirmation. Being encouraged by success in previous cases, physicians at the SKKRC treated this patient with two cycles of LAmB. During his treatment, he only experienced fever with shivering as an adverse event. Investigations before treatment and on follow-up were performed as per previously mentioned cases and all were found within normal or near the normal limit.

\section{DISCUSSION}

All the patients of PKDL were treated with two cycles of LAmB. The dose of each cycle was $20 \mathrm{mg} / \mathrm{kg}$ and was given through four divided doses on every alternate day. The second cycle was given 28 days after the first cycle considering the bioavailability and half-life of the drug. In all the four cases discussed previously, we gave a second cycle of LAmB after microscopy and PCR of the skin sample were positive for Leishmania parasite. The absence of Leishmania donovani (LD) bodies in the microscopy and the negative PCR results for LD body after the second cycle suggest the need for an extended dose schedule for the treatment of nodular PKDL stronger.

The treatment of PKDL is still considered as a gray area of leishmaniasis control and research. ${ }^{3}$ The use of SSG for treatment has been abandoned because of its long course, poor compliance and most importantly, life-threatening adverse events. ${ }^{3}$ The World Health Organization recommends the use of MF for 12 weeks as the first line of treatment. However, the criterion of cure with this drug can only be evaluated at a 12-month follow-up visit with the disappearance of the lesion. Furthermore, it has considerable side effects of vomiting, weakness, nausea, and anorexia. ${ }^{2,7} \mathrm{~Pa}-$ tients with widespread lesions may become noncompliant because of prolonged treatment course, resulting in incomplete cure. Similarly, a large number of patients following the second line of treatment, short course (one cycle) LAmB therapy, have also left treatment with an incomplete cure status. $^{2}$

As PKDL lesions do not interfere with the performance of daily activities, patients usually avoid seeking treatment until the disease has progressed from macular/papular lesions to nodular/ulcerative lesions. At this point of disease progress, patients feel socially stigmatized and seek a cure. Delayed treatment contributes toward spreading VL in endemic communities, especially because human is the only reservoir in this region. The primary goal of treating PKDL patients is to lessen the burden of $\mathrm{VL}$ human reservoirs in the community ${ }^{3}$ but current treatment options are failing to achieve this. Thereby, it is imperative that a treatment option is identified and evaluated that can provide a quick, complete cure. Our recommendations are that a grading system should be devised for PKDL diagnosis based on the intensity and the type of lesions. Based on the grading of the disease, different management options should be listed. The cases highlighted in this report explore the success of using extended LAmB dose schedule for treating a patient with widespread lesions. Moreover, all the cases had minimal or no side effect which should be considered as a unique achievement of this treatment schedule. The success of this alternate treatment schedule for PKDL needs to be supported with clinical trials that evaluate its efficacy and safety.

The presence of good referral systems and planned program activities have made the goal of eliminating PKDL within South East Asia, especially in Bangladesh, a target within reach. It is vital that the number of human reservoirs is controlled to achieve this target. ${ }^{9}$ With the identification of an effective treatment modality of PKDL, the region can make tremendous strides in eliminating PKDL.

Ethical considerations. Written informed consent was taken from all the patients.

Received August 2, 2016. Accepted for publication July 4, 2017.

Published online August 14, 2017.

Acknowledgments: We are thankful to Sumaira Hussain from the Centre for Clinical Epidemiology and Biostatistics (CCEB), School of Medicine and Public Health, Faculty of Health and Medicine, The University of Newcastle (UoN), New South Wales (NSW), Australia, and Tabassum Rahman from Social Research Centre, Victoria, Australia, for their valuable inputs and for improving the language quality of the article. The American Society of Tropical Medicine and Hygiene (ASTMH) assisted with publication expenses.

Financial Support: There was no financial support for conducting the activity.

Authors' addresses: Ariful Basher, Proggananda Nath, Muhammod Abdul Mukit, and Azim Anwarul, Infectious and Tropical Medicine Department, Mymensingh Medical College Hospital (MMCH), Mymensingh, Bangladesh, E-mails: ariful.dr@gmail.com, progganath@yahoo.com, mukit.pharma@gmail.com, and azimanwarul@gmail.com. Shomik Maruf, Fatima Aktar, Rupen Nath, and Dinesh Mondal, Nutrition and Clinical Services Division (NCSD), International Centre for Diarrhoeal Disease Research, Bangladesh (icddr,b), Dhaka, Bangladesh, E-mails: shomik_stj@yahoo.com, fatimaaktar61@gmail.com, rupennath77@ gmail.com, and din63d@icddrb.org. Md Golam Hasnain and Abul Hasnat Milton, Centre for Clinical Epidemiology and Biostatistics (CCEB), School of Medicine and Public Health, Faculty of Health and Medicine, The University of Newcastle (UoN), Newcastle, Australia, E-mails: mdgolam.hasnain@uon.edu.au and milton.hasnat@newcastle.edu.au. AFM Akhtar Hossain, National Kala-azar Elimination Program (NKEP), Directorate General Health Services (DGHS), Ministry of Health and Family Welfare (MoHFW), Government of Bangladesh (GoB), Dhaka, Bangladesh, E-mail: afmhossain@yahoo.com. Abul Khair Mohammad Shamsuzzaman, Disease Control Unit, Directorate General Health Services (DGHS), Ministry of Health and Family Welfare (MoHFW), Government of Bangladesh (GoB), Dhaka, Bangladesh, E-mail: ariful.dr@gmail.com. Ridwanur Rahman, Department of Medicine, Shaheed Suhrawardi Medical College and Hospital (SSMCH), Dhaka, Bangladesh, E-mail: ridwanur@yahoo.com. M. Abul Faiz, Dev Care Foundation, Dhaka, Bangladesh, E-mail: drmafaiz@gmail.com.

\section{REFERENCES}

1. Hasnain MG, Shomik MS, Ghosh P, Rashid MO, Hossain MS, Hamano S, Mondal D, 2016. Case report: post-kala-azar dermal leishmaniasis without previous history of visceral leishmaniasis. Am J Trop Hyg 95: 1383-1385.

2. WHO, 2012. Post Kala-azar Dermal Leishmaniasis: A Manual for Case Management and Control [Internet]. A report of a WHO consultative meeting, Kolkata, India, July 2-3 2012. Available at: http://apps.who.int/iris/bitstream/10665/78608/1/ 9789241505215_eng.pdf Accessed July 16, 2016.

3. Mondal D, Hamano S, Hasnain MG, Stocker AR, 2014. Challenges for management of post kala-azar dermal leishmaniasis and future directions. Res Rep Trop Med 5: 105-111.

4. Molina R, Ghosh D, Carrillo E, Monnerat S, Bern C, Mondal D, Alvar J, 2017. Infectivity of post-kala-azar dermal leishmaniasis patients to sand flies: revisiting a proof of concept in the context 
of the kala-azar elimination program in the Indian subcontinent. Clin Infect Dis 65: 150-153.

5. Mondal D, Nasrin KN, Huda MM, Kabir M, Hossain MS, Kroeger A, Thomas T, Haque R, 2010. Enhanced case detection and improved diagnosis of PKDL in a kala-azar endemic areas in Bangladesh. PLoS Negl Trop Dis 4: e832.

6. National guideline for kala-azar case management, 2015. Kala-azar Elimination Program. Communicable Disease Control. Disease control unit. Directorate general health services. Ministry of health and family welfare. Bangladesh: Government of Bangladesh.

7. Ramesh V, Ansari NA, Jain RK, Salotra P, 2007. Oral miltefosine in the treatment of post-kala-azar dermal Leishmaniasis. Clin Exp Dermatol 33: 103-104.

8. Ramesh V, Singh R, Avishek K, Verma A, Deep DK, Verma S, Salotra $P, 2015$. Decline in clinical efficacy of oral miltefosine in treatment of post kala-azar dermal leishmaniasis (PKDL) in India. PLoS Negl Trop Dis 9: e0004093.
9. Hasnain MG, Basher A, Nath P, Ghosh P, Hossain F, Hossain MS, Mondal D, 2016. Polymerase chain reaction in the diagnosis of visceral leishmaniasis recurrence in the setting of negative splenic smears. Am J Trop Hyg 94: 99-101.

10. Subhashree AR, Parameaswari PJ, Shanthi B, Revathy C, Parijatham BO, 2012. The reference intervals for the haematological parameters in healthy adult population of Chennai, southern India. J Clin Diagn Res 6: 1675-1680.

11. Diana Nicoll C, 2007. Appendix: therapeutic drug monitoring and laboratory reference ranges. Stephen JM, Maxine AP, eds. Current Medical Diagnosis and Treatment, 46th edition. Columbus, OH: McGraw Hill, 1767-1775.

12. Bekersky I, Fielding RM, Dressler DE, Lee JW, Buell DN, Walsh TJ, 2002. Pharmacokinetics, excretion, and mass balance of liposomal amphotericin B (AmBisome) and amphotericin B deoxycholate in humans. Antimicrob Agents Chemother 46: 828-833. 